\title{
Akteur der Sozialwirtschaft und der Zivilgesellschaft
}

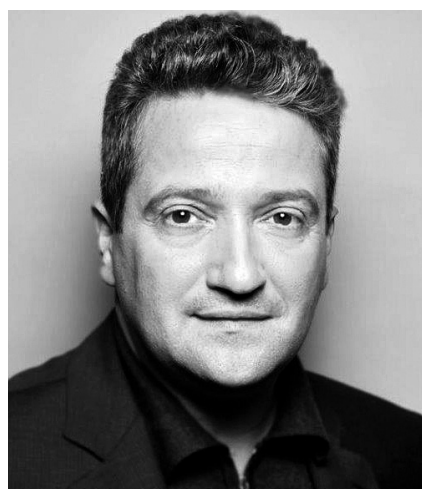

VON THOMAS BEYER

Prof. Dr. iur. Thomas Beyer hat eine Professur für Recht in der Sozialen Arbeit mit den Schwerpunkten Sozialwirtschaft, Recht der Bildung, Recht der Inklusion an der Sozialwissenschaftlichen Fakultät der Technischen Hochschule Nürnberg inne. Seit 2004 ist er ehrenamtlicher Landesvorsitzender der Arbeiterwohlfahrt in Bayern, seit 2012 Vizepräsident des AWO-Bundesverbandes. Zu seinen aktuellen Veröffentlichungen zählen Rechts- und Praxisfragen des Ehrenamtes.

thomas.beyer@th-nuernberg.de

\author{
Seit rund zwei Jahrzehnten gibt es starke \\ marktwirtschaftliche Elemente in der Erbringung \\ sozialer Dienste und beim Betrieb sozialer \\ Einrichtungen. Die Freie Wohlfahrtspflege soll die \\ Diskussion darüber verstärken, welche Folgen diese \\ Entwicklungen hatten - für die Menschen, für die \\ Beschäftigten und für ihr Selbstverständnis als Teil \\ der Sozialwirtschaft wie der Zivilgesellschaft.
}

Der Ausgangspunkt gilt vielen als »Sternstunde« des Sozialstaates: die Einführung der Sozialen Pflegeversicherung durch das Pflege-Versicherungsgesetz vom 26. Mai 1994. Tatsächlich verbindet sich mit diesem vermeintlichen Höhepunkt der Ausgestaltung des deutschen Sozial-(Versicherungs-)systems ein doppelter Paradigmenwechsel.

"Zum Ausgleich der mit den Arbeitgeberbeiträgen verbundenen Belastungen der Wirtschaft« (\$S 58 Abs. 2 SGB XI) vollzieht sich der Einstieg in die Abkehr vom Prinzip der Parität der Finanzierung der Gesetzlichen Sozialversicherungen durch Arbeitgeber und Arbeitnehmer sinnfällig in einer ausdrücklich höheren Beitragslast der Beschäftigten in den Ländern der Bundesrepublik Deutschland, in denen man auf die Kompensationsmaßnahme einer Streichung des Bußund Bettages als gesetzlichem Feiertag verzichtet ( $\int 58$ Abs. 3 SGB XI).

Tiefgreifend gerät der Systemwechsel für die Freien Träger. Für sie bedeutet die Einführung der Pflegeversicherung die Ablösung der bis dahin bestehenden Strukturen für Leistungserbringung und Finanzierung. Die Stichworte lauten: Wirtschaftlichkeitsgebot und Wirtschaftlichkeitsprüfung (SS 29, 79 SGB XI), Drittvergleich bei den Kosten im Rahmen der Pflegesatzvereinbarung ( $\int 84$ Abs. 2 S. 7 SGB XI), Abkehr von der bedarfsdeckungsorientierten Refi- nanzierung hin zum Instrument der Leistungsvereinbarung ( $\int 85$ SGB XI), also einem Vertragsverhältnis auf Gegenseitigkeit mit ausdrücklicher Zuweisung des Unternehmerrisikos an den Einrichtungsträger ( $\int 84$ Abs. 2 S. 5 SGB XI). Begleitend erfolgt die Gleichstellung, ja die Forcierung des Markteintritts privater Anbieter gegenüber gemeinnützigen Einrichtungsträgern, insbesondere der Freien Wohlfahrtspflege ( $\mathbb{S} 11$ Abs. 2 S. 3, 72 Abs. 3 S. 2 SGB XI).

In der Folge vollzieht sich die Übertragung dieser Entwicklungen auf die Kinder- und Jugendhilfe (vgl. $\mathbb{S} \int 78$ a ff. SGB VIII) und die Sozialhilfe (\$S 75 ff. SGB XII). Mit der Gleichstellung und Neuzulassung (vgl. \$74 a SGB VIII) privater Leistungserbringer im Bereich der Kindertageseinrichtungen in Folge des Tagesbetreuungsausbaugesetzes (TAG) von 2004 sowie des Kinderförderungsgesetzes (KiföG) von 2008 unterstehen im Jahr 2014 alle wesentlichen Bereiche der Leistungserbringung in der Sozialen Arbeit einer marktwirtschaftlichen Rahmensetzung.

Die Konsequenzen für die gemeinnützige Wohlfahrtspflege sind tiefgreifend: gesellschaftsrechtliche Ausgliederungen aus der vordem überwiegend vereinsmäBig organisierten Trägerstruktur; der nicht nur damit verbundene - Rückgang der Tarifbindung allgemein sowie Strukturveränderungen bei den Arbeitsver- 
tragsrichtlinien der kirchlichen Wohlfahrtsverbände; daraus resultierend der Vorwurf einer »Tarifflucht « nicht nur seitens der Beschäftigten, ihrer Interessenvertretungen sowie der Öffentlichkeit; eine oft kritiklose Übernahme ausschließlich wirtschaftlichkeitsorientierter Denkmuster von der "privaten Konkurrenz«, nicht selten verbunden mit dem Argument, man sei sonst doch gezwungen, weiteres Terrain an diese aufzugeben.

Dessen ungeachtet - oder vielleicht auch gerade deswegen - existiert die Behauptung einer "Privilegierung « der Freien Wohlfahrtspflege fort und sei es oft auch nur in einer auffälligen Verteidigungshaltung ihrer Vertreter. Tatsächlich hätte sich solchen Angriffen auf die Gemeinnützigkeit schon von jeher strukturell deren Verknüpfung mit zwingenden Bindungen für die Führung sozialwirtschaftlicher Unternehmen entgegenhalten lassen. Die Steuervergünstigung korrespondiert und begründet sich anhand von Bindungen, die der Ausübung der freien unternehmerischen nach vorgeblich verbliebenen "Reservaten ", dabei sowohl die fachlichen und rechtlichen Maßstäbe der »anderen Aufgaben « der Jugendhilfe ( $\mathbb{S} \int 2$ Abs. 1 und 76 Abs. 1 SGB VIII) verkennend wie das tatsächlich überhaupt nicht gegebene Interesse privater Anbieter verschweigend, hierfür personelle und finanzielle Ressourcen aufzuwenden (vgl. Boeßenecker/Vilain 2013, 63).

Ziel ist also etwas anderes: Der öffentlich bekundete "Anfangsverdacht " einer Marktbeeinträchtigung durch die gesetzlich vorgegebenen Organe der Jugendhilfeplanung soll diese im Sinne einer gewollten weiteren Privatisierung der Jugendhilfebelange beeinflussen.

\section{Folgenlose Klagen}

Wer hat's erfunden? Der Gesetzgeber des Pflege-Versicherungsgesetzes bringt den gewünschten neuen Primat der Ökonomie in der Sozialen Arbeit fast schon überdeutlich zum Ausdruck, wenn er Pflegeeinrichtungen allen fachlichen

\section{"Die Freie Wohlfahrtspflege muss selbst in die Offensive gehen"}

Tätigkeit auch im Sozialen Bereich sonst völlig fremd sind (Beyer 2010, 251 f.). In Bezug auf die Leistungserbringung garantiert der Rechtsrahmen die Gleichstellung privater Anbieter.

20 Jahre marktwirtschaftliches System im sozialen Bereich haben aber auch auf gewachsene und begründete fachliche Funktionsvorbehalte der gemeinnützigen Wohlfahrtspflege mehr und mehr Zugriff entwickelt - sei es im Bereich Ehrenamt und Freiwilliges Engagement oder Tätigkeit als anerkannte Träger der Freien Jugendhilfe. Dass die Monopolkommission derzeit die gesetzlich vorgesehene Mitwirkung der Freien Wohlfahrtspflege im Bereich der Jugendhilfeausschüsse ( $\mathbb{S} 71$ Abs. 1 SGB VIII) thematisiert (Monopolkommission 2014), ist bezeichnend.

Weil ordnungspolitisch gewichtige Ausprägungen einer Vorrangstellung der Freien Wohlfahrtspflege im Leistungsbereich der Jugendhilfe (vgl. $\mathbb{S} 2$ Abs. 2 SGB VIII) ebenso wie auf dem weiteren Sozialmarkt rechtlich nicht (mehr) existieren, begibt man sich auf Spurensuche
Kriterien voran als "selbständig wirtschaftende Einrichtungen « charakterisiert ( $\int 71$ Abs. 1 und 2 SGB XI).

Warum ist es der Freien Wohlfahrtspflege auch 20 Jahre später nicht gelungen, Zielsetzung wie Reichweite dieser klaren Richtungsentscheidung zu verdeutlichen? Ihr Unvermögen oder ihre Scheu, gerade gegenüber der Politik die Verantwortungsbeiträge für die rechtlichen und strukturellen Grundlagen ihrer Arbeit deutlich zu machen, lässt die oft routiniert empfundenen öffentlichen Klagen der Verbände über eine permanente Verschärfung der Rahmenbedingungen für die Soziale Arbeit zunehmend als so wirkungslos erscheinen.

\section{Wenn Wettbewerb, dann richtig!}

Die Freie Wohlfahrtspflege scheint auch 20 Jahre nach der Etablierung marktwirtschaftlicher Strukturen sich ihres Standortes in der Sozialwirtschaft nicht immer sicher zu sein. Dies schließt den Eindruck einer Überanpassung manch- mal nicht aus. Konzernstrukturen wurden geschaffen, die zuweilen mehr Vorbildern der Industrie oder Strukturhandbüchern von Unternehmensberatungen entsprungen schienen als den eigenen tatsächlichen Bedürfnissen. Die Etablierung von Servicegesellschaften samt Tarifwechsel, Leiharbeit nicht nur extern in Anspruch genommen, sondern mittels der eigenen konzerninternen Arbeitnehmerüberlassung - nicht jede teilweise von der Öffentlichkeit äußerst kritisch begleitete Entwicklung innerhalb der verbandlichen Sozialwirtschaft war dabei objektiv nur den neuen Rahmenbedingungen geschuldet.

Ähnliches zeigt sich in der Darstellung der Wohlfahrtspflege nach außen. In dem nachvollziehbaren Bemühen, den erheblichen eigenen Wertschöpfungsbeitrag und die Beschäftigungspotenziale $\mathrm{zu}$ verdeutlichen, wurden seitens der Landesarbeitsgemeinschaften und Ligen der Freien Wohlfahrtspflege umfangreiche Studien zu ihrem volkswirtschaftlichen Beitrag auf den Weg gebracht (vgl. u. a. für Bayern Puch/Schellberg 2010; für Sachsen Karmann et. al. 2011; für Sachsen-Anhalt isw 2012).

So treffend die Untersuchungen die Stellung der Freien Wohlfahrtspflege in der Sozialwirtschaft darstellen konnten, so deutlicher wurde indes, dass die Verbände der Frage - und erst recht der Antwort - nach dem spezifischen »Mehrwert" einer gemeinnützigen Leistungserbringung innerhalb der Sozialwirtschaft weit weniger Beachtung schenkten. Dieser Mangel lässt sich bis heute schmerzhaft empfinden.

\section{Veränderung der Binnenstrukturen}

Die sozialwirtschaftliche Erneuerung hat die Freie Wohlfahrtspflege nicht nur in dem Verhalten nach außen verändert, sondern tiefgreifend auch in ihrer verbandlichen Arbeit. Dies gilt insbesondere für das die Freie Wohlfahrtspflege prägende Element des freiwilligen Engagements. Hierzu konnte an anderer Stelle (Beyer 2012, 29 f.) bereits näher ausgeführt werden: "Das Herausstellen der wirtschaftlichen Funktion der Wohlfahrtspflege wird gerade von den ehrenamtlich Tätigen keineswegs immer nur als Ausdruck einer notwendigen Professionalisierung, sondern durchaus als jetzt gewollte Dominanz eines betriebswirtschaftlichen Blick- 


\section{"Soziales unternehmen: Zwischen Grundsatz und Umsatz"}

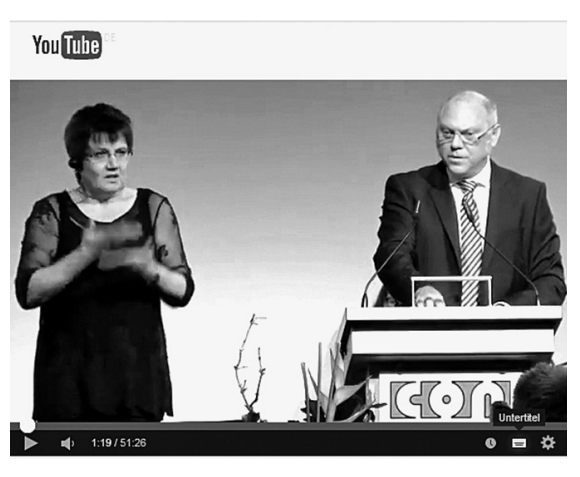

Soziale Dienstleistungen sollten nach Einschätzung des Hauptgeschäftsführers des Paritätischen Wohlfahrtsverbandes, Dr. Ulrich Schneider, nicht länger so stark nach ihrer Wirtschaftlichkeit beurteilt werden. Es sei dringend an der Zeit, nach dem Ökonomisierungsschub in der Sozialen Arbeit wieder das Pendel zurechtzurücken, sagte er bei der ConSozial 2013 in Nürnberg. Stattdessen sollten sich Wohlfahrtsverbände wieder auf traditionelle Wurzeln und Werte besinnen. Von Kran- ken- und Pflegekassen forderte Schneider mehr Vertrauen bei Abrechnungen, um Menschen angemessen helfen zu können. Der Eröffnungsvortrag unter dem Thema "Soziales unternehmen: Zwischen Grundsatz und Umsatz "steht auf dem YouTube-Kanal des Paritätischen zum Anschauen und Nachhören zur Verfügung (http://www.youtube.com/watch?v= izJdOqEXqso).

www.consozial.de winkels verstanden, die dem Ehrenamt immer weniger Raum lässt.

Nahezu flächendeckend hat die Modernisierung der Binnenstruktur der Wohlfahrtsverbände zu einer Entkoppelung von verbandlichem Sektor und Sozialunternehmen geführt. Damit ändert sich auch der Standort des Ehrenamtes. War die Rolle des Ehrenamtes in den Leitungsfunktionen lange unangefochten (...), so kommt es immer öfter zur Trennung von Unternehmensführung und ehrenamtlicher Vorstandsverantwortung im Verband. Mag dies einerseits als Zeichen einer neuen, modernen Unternehmenskultur auch bei sozialen Einrichtungen und Diensten verstanden werden, so geht damit andererseits unverkennbar der Einfluss der ehrenamtlich Engagierten auf die sozialpolitische Orientierung auch des Sozialunternehmens zurück.«

\section{Verlorene Glaubwürdigkeit}

Es zeigt sich eine fatale Wechselwirkung: Die Freie Wohlfahrtspflege verliert an Glaubwürdigkeit und mit ihr die bei ihr ehrenamtlich Engagierten. Die Freie Wohlfahrtspflege verliert an Glaubwürdigkeit, weil sie Ehrenamtliche verliert; und weil sie Ehrenamtliche verliert, verliert sie auch in der öffentlichen Wahrnehmung noch mehr an Glaubwürdigkeit.

In diesem Zusammenhang deutlich wird auch eine strategische Fehleinschätzung der Wohlfahrtsverbände. In ihrer Selbstwahrnehmung wie auch in ihrem Auftreten nach außen propagiert sie unverändert eine Rolle als wesentlicher Akteur bei der Bindung und Gestaltung bürgerschaftlichen Engagements (Beyer 2012, 28 f.). Sie nimmt dabei zu wenig wahr, wie der nicht zuletzt im Rahmen der Politik des »aktivierenden Sozialstaats« der damaligen Bundesregierung zu Beginn der 2000er Jahre (Beyer 2006) bewusst von staatlicher Seite auf den Weg gebrachte Ausbau neuer »zivilgesellschaftlicher « Strukturen (Beyer 2012, 31) der Wohlfahrtspflege mit einem Kernbereich identitätsstiftender Aufgaben zugleich einen Großteil eigenen, prägenden Charakters genommen hat (Dahme/Wohlfahrt 2011, 122 f.).

\section{Vergessene Maßstäbe}

Die Wohlfahrtspflege fragt durchaus, warum sie an öffentlicher Wertschätzung verliert. Sie verdrängt dabei aber, dass sie etwa über die Arbeitgeber-Auffassungen einzelner ihrer Vertreter hinaus grundsätzlich öffentliche Kritik auf sich zieht. Dies deshalb, weil sie als gemeinnützigkeitsverpflichtet strukturell und zu Recht - von der Allgemeinheit an besonderen Maßstäben gemessen wird.

Die Freie Wohlfahrtspflege setzt sich beispielsweise zu diesen Maßstäben - und nicht nur zu ihrer vorherigen lautstarken Forderung nach einer besseren Personalausstattung - in offenen Widerspruch, wenn sie, wie jetzt in Bayern, eine nach Jahren durchgesetzte Verbesserung des Stellenschlüssels in der Pflege (Beschluss der Landespflegesatzkommission Bayern vom 18. Dezember 2013) in der Praxis zum Teil nicht umsetzt und Verantwortliche der operativen Ebene das auch noch in einer die Weltfremdheit der Spitzenverbandsebene ironisierenden Weise öffentlich machen (vgl. Schneider 2014, 10).

Wenn dafür offen die Begründung gegeben wird, höhere Pflegesätze ließen sich »am Markt nicht durchsetzen «, wird nicht nur erneut eine von gewerblichen Überlegungen nicht mehr unterscheidbare Konkurrenzorientierung der Freien Wohlfahrtspflege deutlich. Auffällig ist insbesondere, dass solche Äußerungen den Beleg dafür bieten, dass dort ein Ansatz für ein Wettbewerbsverständnis jenseits des reinen Marktpreises überhaupt nicht mehr für denkbar gehalten wird. Der fast zwingende Schluss der Öffentlichkeit: Trotz aller Bekundungen habe die Wohlfahrtspflege letztlich kein Interesse oder jedenfalls kein Zutrauen mehr zu einem Wettbewerb in der Sozialen Arbeit über besondere Qualitätsmerkmale.

Ein darin liegender Widerspruch zu den behaupteten Alleinstellungsmerkmalen der Freien Wohlfahrtspflege etwa aus christlichen oder soziokulturellen Bezügen verschärft deren Glaubwürdigkeitskrise weiter. Ein Versagen der Anwaltsfunktion der Wohlfahrtsverbände (Beyer 2010, 249) tritt hinzu. Der permanent als unangemessen kritisierte Zwang zu einer Pflege zum »Billig-Tarif « ist letztlich so verinnerlicht, dass man deren Überwindung als nicht einmal mehr denkbar ansieht. Wäre es nicht Aufgabe der Wohlfahrtspflege, eine Debatte um das, "was gute Pflege kosten darf - ja muss « aktiv gegenüber den ökonomischen Interessen aller Beteiligten einzufordern?

\section{Vergebene Chancen}

Die Fixierung auf den Marktpreis als verengte Interpretation des Wettbewerbsgedankens lässt gerade Verantwortliche der Wohlfahrtsverbände fast reflexartig Position beziehen, selbst gegen innovative Versuche, durch ordnungspolitisch angemessene Instrumente die Voraussetzungen für einen fairen Wettbewerb erst zu schaffen.

Deutlich zeigt sich dies am teilweise massiven Unverständnis, mit dem innerhalb der Wohlfahrtspflege anfangs dem Vorstoß für einen allgemein-verbindlichen Tarifvertrag Soziales und für die Pflege (Arbeiterwohlfahrt Bundeskon- 


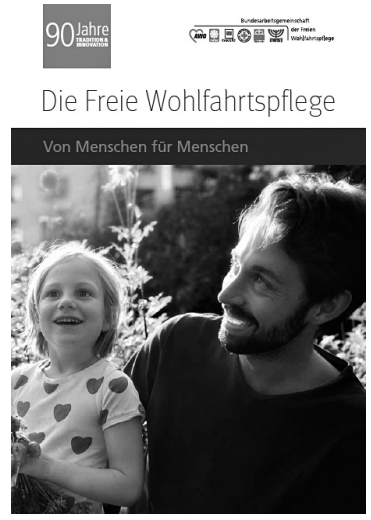

Die neue Imagebroschüre der Bundesarbeitsgemeinschaft der Freien Wohlfahrtspflege e. V. ist fertig. Die farbige Publikation stellt die Grundsätze und die volkswirtschaftliche Bedeutung, die Arbeitsfelder und die Angebote der Mitgliedsorganisationen der Freien Wohlfahrtspflege vor. Die 28-seitige Broschüre kann von der Website der Organisation heruntergeladen werden und auch kostenlos als Druckexemplar, beispielsweise zur Auslage in Dienststellen oder bei Veranstaltungen, angefordert werden.

www.bagfw.de/de/spezialseiten/ artikeldetail/ueber_uns

ferenz 2012; Arbeiterwohlfahrt Landesverband Bayern 2012) begegnet wurde. Die Angst, mit den eigenen Kosten im eng umkämpften regionalen Markt nicht mehr mithalten zu können, nimmt dann den Blick darauf, dass dieses gesetzlich lange eingeführte tarifrechtliche Instrument ( $\mathbb{S} 5 \mathrm{TVG}$ ) gerade zu einer gleichmäßigen Anlastung von Personalkosten auch bei solchen Anbietern führen würde, die die Vorteile ihrer nicht gegebenen Tarifbindung unmittelbar betriebswirtschaftlich umsetzen, bis hin zu einer »Flexibilisierung « der Gehälter stark umworbener Fachkräfte.

\section{Ein Modell für das 21. Jahrhundert}

Die Verbände tragen mit ihren sozialwirtschaftlichen Organisationen heute wenig bei zu einer schlüssigen Darlegung der Spezifika einer gemeinnützigen Freien Wohlfahrtspflege. Wohlgemerkt: Es geht nicht um eine verklärende Sicht auf die Zeiten der Bedarfsdeckungsfinanzierung. Es drängt sich aber der Eindruck auf, als ob gerade die operative Ebene der Wohlfahrtspflege oftmals überhaupt nicht mehr den Glauben an eine Eingrenzung des puren Wettbewerbsgedanken in der Sozialen Arbeit zu entwickeln vermag. Sie konterkariert so die gegensätzlichen Bemühungen der Verbandsspitzen in der Öffentlichkeit (www.Buendnis-fuergute-Pflege.de). Gravierender: Sie negiert die längst eingesetzte Rückbesinnung der öffentlichen Meinung hin zu einer Zurückweisung rein marktlicher Prinzipien gerade im Bereich der öffentlichen und sozialen Daseinsvorsorge.

In einer Zeit, in der private Geschäftsbanken nach der Weltwirtschafts- und Finanzkrise mit "neuer Verantwortung « werben, in einer Zeit, in der Privatisierungsabsichten hinsichtlich der öffentlichen Wasserversorgung oder die Aushandlung von Freihandelsabkommen breite und engagierte öffentliche Debatten auslösen, ergibt sich ausgerechnet die Freie Wohlfahrtspflege scheinbar resigniert ihrem Schicksal.

Gerade auf dem Markt aber gilt, dass nur das Verstehen, das Herausarbeiten und die konsequente Pflege eines Alleinstellungsmerkmals dauerhaft die eigene Wettbewerbsposition zu sichern vermag. Die Freie Wohlfahrtspflege muss selbst in die Offensive gehen, um ihre Strukturen und Qualitätsmerkmale, ihren Mehrwert zu verdeutlichen - nicht zu rechtfertigen.

Ansatzpunkte hierfür gibt es vielfältig. So lässt sich etwa zeigen, dass gerade in Verbindung mit der Gemeinnützigkeit die Freie Wohlfahrtspflege strukturell über Merkmale einer guten Unternehmensführung verfügt, über die andere Wirtschaftsbereiche in Good Governance-Kommissionen erst nachzudenken beginnen (Beyer 2010, $251 \mathrm{f}$.).

Es ist Zeit für die Freie Wohlfahrtspflege, ein Selbst-Bewusstsein im wahrsten Sinne des Wortes wieder zu entwickeln. Die Geschichte der Wohlfahrtspflege ist nicht neu zu schreiben. Anliegen wie Interesse der Praxis sollte es aber sein, eine breite gesellschaftliche und wissenschaftliche Debatte anzustoßen, wie sich ein konsistentes Modell Freie Wohlfahrtspflege für das 21. Jahrhundert darstellen und tatsächlich leben lässt.

20 Jahre nach dem marktwirtschaftlichen Systemwechsel im sozialen Bereich mag darin der erfolgversprechende Ansatz für die Freie Wohlfahrtspflege liegen, endlich ihren Platz im Wettbewerb der Sozialen Arbeit zu verstehen; als Akteur der Sozialwirtschaft gleichermaßen wie der zivilgesellschaftlichen Selbstverantwortung. Die Zeit, sich vermeintlich für das eine oder das andere entscheiden zu können - für die Verbände der Freien Wohlfahrtspflege ist sie vorbei.

\section{Literatur}

Arbeiterwohlfahrt Bundeskonferenz 2012,

Beschluss zu Antragsnummer 2.1.-107, Eckpunkte für wertegebundene AWO Unternehmen. Arbeiterwohlfahrt Landesverband Bayern, AWO geht in die Offensive für einen allgemeinverbindlichen Branchentarifvertrag Soziales, Pressemitteilung vom 3. Januar 2012.

Beyer, Thomas, Der "aktivierende« Sozialstaat ein passiver Staat, Bayerische Sozialnachrichten 2/2006, S. 11.

Beyer, Thomas, Identität statt Ressource, Das Ehrenamt und die Freie Wohlfahrtspflege, in: Rosenkranz, Doris/Weber, Angelika (Hg.), Freiwilligenmanagement, Einführung in das Management von Ehrenamtlichen in der Sozialen Arbeit, Zweite Auflage, Weinheim und Basel 2012, S. 27-34

Beyer, Thomas, Zukünftiger Stellenwert von Unternehmen der Freien Wohlfahrtspflege im Bereich der Sozial- und Gesundheitswirtschaft, in: Horneber, Markus/Helbich, Peter/Raschzok, Klaus (Hg.), Dynamisch Leben gestalten, Perspektiven Zukunftsorientierter Unternehmen in der Sozial- und Gesundheitswirtschaft, Stuttgart 2010, S. 243-255.

Boeßenecker, Karl-Heinz/Vilain, Michael, Spitzenverbände der Freien Wohlfahrtspflege. Eine Einführung in Organisationsstrukturen und Handlungsfelder sozialwirtschaftlicher Akteure in Deutschland, Zweite Auflage,

Weinheim und Basel 2013.

Dahme, Heinz-Jürgen/Wohlfahrt, Norbert, Freie Wohlfahrtspflege und Bürgerschaftliches Engagement - eine Zwischenbilanz, TuP 2011, S. 115-124. isw Institut für Strukturpolitik und Wirtschaftsförderung gGmbH, Sozialwirtschaft Sachsen-Anhalt, Studie im Auftrag der LIGA der Freien Wohlfahrtspflege im Land SachsenAnhalt e.V., Magdeburg 2012.

Karmann, Alexander et. al., Gutachten zur Sozialwirtschaft in Sachsen unter besonderer Berücksichtigung der Freien Wohlfahrtspflege, Dresden 2011.

Monopolkommission, Anhörung zur Vorbereitung des XX. Hauptgutachtens gemäß $\$ 44$ Abs. 1 Satz 1 GWB, Fragebogen, Bonn 2014. Puch, Hans-Joachim/Schellberg, Klaus, Sozialwirtschaft Bayern, Umfang und wirtschaftliche Bedeutung, Nürnberg 2010.

Schneider, Kerstin, Alle wollen mehr Personal, aber wer kann es bezahlen? Verbesserter Stellenschlüssel in Bayern stellt Einrichtungen vor Herausforderungen, Altenheim 2014, S. 10. 